\title{
Avaliação das estatísticas utilizadas nos controles de qualidade de equipamentos para radiodiagnóstico
}

Evaluation of the statistics used in the quality control of equipment for radiodiagnostic

\author{
A. S. A. Santana ${ }^{1 *}$; M. V. T. Navarro ${ }^{2}$; R. R. Lima ${ }^{1}$ \\ ${ }^{1}$ Programa de Pós-graduação em Estatística e Experimentação Agropecuária, Universidade Federal de Lavras, CEP: \\ 37200-000, Lavras, Minas Gerais, Brasil \\ ${ }^{2}$ Departamento de Ciências Básicas, Instituto Federal de Educação, Ciência e Tecnologia da Bahia, CEP: 40302-015, \\ Salvador-Bahia, Brasil.
}

*azly@ifba.edu.br

(Recebido em 22 de outubro de 2015; aceito em 05 de fevereiro de 2016)

\begin{abstract}
O objetivo deste estudo foi verificar e analisar a adequação das estatísticas utilizadas em metodologias nos testes de controle de qualidade para estimação da exatidão e precisão das variáveis de tensão no tubo e tempo de exposição. Foram verificadas as metodologias recomendadas na Portaria Federal 453/98, no Guia Radiodiagnóstico Médico - Segurança e Desempenho de Equipamentos publicado pela Anvisa, o Protocolo Español de Control de Calidad em Radiodiagnóstico e Protocolos de Control de Calidad em Radiodiagnóstico. A adequação das estatísticas foi baseada nas definições do Vocabulário Internacional de Metrologia e em conceitos estatísticos. Além disso, elas foram comparadas entre si considerando o percentual de não conformidade (ou rejeição) de equipamentos apresentado pela metodologia aplicada. Nessa última avaliação foram utilizados dados simulados com o objetivo de obter distribuições empíricas. Foram encontradas diferenças entre as estatísticas e tolerâncias indicadas para as avaliações de precisão, feitas por meio dos testes de reprodutibilidade e/ou repetitividade, e nas avaliações da exatidão da tensão no tubo e tempo de exposição. Apesar de estarem em acordo com as definições estatísticas e metrológicas, as diferentes estatísticas apresentadas, conduziram distintos percentuais de não conformidade dos equipamentos para o mesmo tipo de avaliação, apontando estatísticas com maior sensibilidade na detecção de valores discrepantes, o que leva à reprovação de um equipamento de radiodiagnóstico. Tolerâncias diferentes também influenciaram na porcentagem de reprovação e levaram a resultados diferentes para um mesmo equipamento. Dessa forma, pode-se obter diferentes laudos de conformidade de um equipamento, conduzindo a resultados inconsistentes.

Palavras-chave: exatidão, repetitividade, reprodutibilidade
\end{abstract}

The objective of this study was to verify and analyze the adequacy of the statistics used in methodologies on quality control tests for the estimation of accuracy and precision of the tube voltage and exposure time variables. The methodologies recommended in the Portaria Federal 453/98, in the Guia Radiodiagnóstico Médico - Segurança e Desempenho de Equipamentos published by Anvisa, the Protocolo Español de Control de Calidad em Radiodiagnóstico and Protocolos de Control de Calidad em Radiodiagnóstico. The adaptation of the statistics was based on the definitions of the International Vocabulary of Metrology and on statistical concepts. In addition, they were compared between each other considering the percentage of non-conformity (or rejection) of equipment presented by the applied methodology. In this last evaluation, simulated data were used with the objective of obtaining empirical distributions. Differences were found between the statistics and tolerances indicated for the precision evaluation, performed by means of reproducibility and/or repetitiveness tests and, in the accuracy evaluations, in the tension of the tube and exposure time. Despite being in accordance with the statistical and metrological definitions, the different statistics presented conducted distinct percentages of non-conformity in the equipment for the same type of evaluation, pointing to statistics with higher sensitivity in the detection of discrepant values, which leads to the disapproval of a radiodiagnostic equipment. Different tolerances also influenced the disapproval percentage and led to different results for the same equipment. Thus, different conformity reports might be obtained of an equipment, leading to inconsistent results.

Keywords: accuracy, repeatability, reproducibility 


\section{INTRODUÇÃO}

No início da década de 1980, com a consolidação do conceito de qualidade em radiodiagnóstico, como fundamental no processo de controle dos riscos, a Organização Mundial de Saúde publicou a primeira recomendação internacional intitulada Quality Assurance in Diagnostic Radiology [1]. Essa recomendação estabeleceu que os principais objetivos de controle, em serviços de radiodiagnóstico, deveriam visar o diagnóstico correto, a redução de dose e dos custos dos serviços. Além disso, instituiu recomendações que visavam incentivar e orientar as autoridades reguladoras, das comissões internacionais e dos serviços, no sentido de melhorar a efetividade dos radiodiagnósticos, oferecendo as bases teóricas e operacionais para a regulação na área.

Em 1997, a Organização Mundial de Saúde juntamente com a Organización Panaamericana de la Salud publicou um documento intitulado Organización, Desarrollo. Garantía de calidad y radioprotección en los servivios de radiología: imaginología y radioterapia [2] onde ilustra aspectos tais como a garantia e controle de qualidade em radioproteção.

No Brasil, o processo de implantação da regulamentação, estabelecendo a obrigatoriedade de programas de garantia de qualidade em radiodiagnóstico, teve início no Estado de São Paulo com a publicação da Resolução 625/94 [3], em 14 de dezembro de 1994, que ampliaria para todo o país em 1998, através da Portaria Federal MS SVS 453/98 [4, 5].

A Portaria Federal MS SVS 453/98 [4] exigia que todos os serviços de radiodiagnóstico médico e odontológico implementassem um programa de garantia de qualidade, incluindo os testes de controle de qualidade. Esse controle deveria ser feito por meio de testes de controle de qualidade que avaliam o equipamento, pela verificação de desempenho de diversos parâmetros técnicos que influenciam na qualidade da imagem, tais como exatidão e precisão do tempo de exposição e tensão no tubo de imagem. Esses testes deveriam ser feitos de forma regular e periódica, e deveriam estar de acordo com os critérios indicados pelo protocolo adotado.

Entretanto, apesar da Portaria Federal MS SVS 453/98 indicar os teste de controle de qualidade e estabelecer limites de aceitação e frequência, a mesma não trata dos procedimentos para execução. Nesse sentido, a Agência de Vigilância Sanitária (ANVISA), fundamentada na Portaria e normas internacionais, publicou a resolução $\mathrm{N}^{\circ} 64$ de 4 de abril de 2003 [6], na qual aprovou o Guia Radiodiagnóstico Médico: Segurança e Desempenho de Equipamentos [7], como uma publicação complementar ao disposto na Portaria Federal MS SVS 453/98.

Tal guia, descreve os procedimentos para os testes propostos e outros não incluídos na Portaria Federal MS SVS 453/98 para cada tipo de equipamento (raios X convencionais, mamográficos, fluoroscopia e tomógrafos computadorizados) com o objetivo de contribuir na uniformização dos procedimentos para realização dos testes de qualidade nos equipamentos de radiodiagnóstico médico. Até então, era comum os especialistas seguirem as recomendações de organismos internacionais, dentre eles o Protocolo Español de Control de Calidad em Radiodiagnóstico (PE) [8] e Protocolos de Control de Calidad em Radiodiagnóstico (ARCAL XLIX) [9].

Com a comparação da Portaria Federal MS SVS 453/98 [4] com os demais documentos mencionados, verifica-se que não existe uniformidade nas estatísticas utilizadas nas avaliações da exatidão e precisão de variáveis como tensão no tubo de imagem e tempo de exposição. Portanto, deve-se verificar se o uso de diferentes estatísticas pode levar a diferenças na conclusão sobre conformidade ou não, de um equipamento de radiodiagnóstico em teste.

A acurácia e precisão são termos comuns nos campos da Metrologia e Estatística, utilizadas no controle de qualidade em radiodiagnóstico. A acurácia no campo da Metrologia é comumente chamada de exatidão, e a precisão é uma característica verificada por meio de testes de reprodutibilidade e/ou repetitividade.

No contexto do controle de qualidade de equipamentos para radiodiagnóstico, a reprodutibilidade e/ou repetitividade objetivam avaliar o desempenho do equipamento por meio das dispersões das medidas geradas por esses equipamentos. No entanto, estes testes também são aplicados em laboratórios analíticos, por meio de comparações interlaboratoriais, em controle de qualidade de procedimentos como descreve o trabalho de Campos et al. [10] e Chui et al. [11] ou como parâmetro para avaliar metodologias para teste de germinação em sementes de nabo forrageiro visto no artigo de Kataota et al. [12]. 
As avaliações recomendadas nos Programas de Garantia de Qualidade em Radiodiagnóstico, que consideram tais termos, devem estar em consonância com os objetivos requeridos pela área, com as definições apresentadas no Vocabulário Internacional de Metrologia (VIM) [13] e com os conceitos estatísticos.

Conforme o Vocabulário Internacional de Metrologia, exatidão (ou acurácia) é o grau de concordância entre o valor medido e um valor verdadeiro de um mensurando. Este valor verdadeiro na prática do controle de qualidade em radiodiagnóstico é interpretado pelo valor nominal (Yn) e o valor medido (Yi), é o valor gerado pelo equipamento em teste e observado por um instrumento de medida, devidamente calibrado, conforme normas estabelecidas.

As avaliações de exatidão e precisão são feitas considerando expressões que estão em função dos dados amostrais ou valores observados. Segundo Ferreira [14], expressões com estas características são chamadas de estatísticas ou estimadores.

Os valores gerados por essas expressões são ditas estimativas e ao serem comparadas com as tolerâncias estabelecidas nas publicações indicarão se o equipamento está ou não em conformidade, em relação ao protocolo de referência. Estatísticas que apontem possíveis valores discrepantes mostram-se mais adequadas, considerando que esses valores acarretarão um aumento prejudicial de dose no paciente ou uma diminuição, que influenciará na qualidade da imagem.

Assim, este trabalho tem como objetivo identificar e comparar as estatísticas consideradas na avaliação de equipamentos de radiodiagnóstico, discutir a adequação com as definições metrológicas e verificar se as diferentes metodologias de cálculo, propostas na Portaria Federal MS SVS 453/98 e demais protocolos, podem gerar discordâncias nos resultados.

\section{MATERIAL E MÉTODOS}

As estatísticas (ou estimadores) indicadas na Portaria Federal MS SVS 453/98, no Radiodiagnóstico Médico: Segurança e Desempenho de Equipamentos, no Protocolo Español de Control de Calidad em Radiodiagnóstico 2011 e no Protocolos de Control de Calidad em Radiodiagnóstico, para avaliação da precisão, por meio de teste de reprodutibilidade e repetitividade, e da exatidão da tensão no tubo e do tempo de exposição, foram identificadas e comparadas.

A partir das definições do Vocabulário Internacional de Metrologia e das características dos estimadores indicados nas publicações, foram analisadas a adequação das várias estatísticas recomendadas nas avaliações dos equipamentos de radiodiagnóstico.

Para a comparação das diferentes estatísticas, inicialmente foi realizado o levantamento de todas as estatísticas indicadas, bem como as respectivas tolerâncias, para os equipamentos utilizados em mamografias e radiografias. Além disso, como forma de identificar existência de discrepâncias com o uso de diferentes estatísticas, na avaliação de um mesmo equipamento, foram utilizados dados simulados. Na simulação, foram consideradas diferentes situações ou cenários, os quais englobavam basicamente vários conjuntos de observações com a presença de valores mais ou menos discrepantes do valor nominal do equipamento.

As simulações foram feitas utilizando-se o software R [15], considerando-se diferentes valores nominais para tensão (indicados em $\mathrm{kVp}$ ) e tempo de exposição (indicados em ms) geralmente utilizadas clinicamente em serviços de mamografia e radiografia. Para avaliações de exatidão em mamógrafos, foram considerados valores nominais de tensão iguais a $20 \mathrm{kVp}, 25 \mathrm{kVp}$ e $30 \mathrm{kVp}$. Para tempo de exposição os valores foram $100 \mathrm{~ms}, 500 \mathrm{~ms}$ e $1.000 \mathrm{~ms}$. Para os equipamentos utilizados em radiografia, ou seja, os de raios $\mathrm{X}$ convencionais, foram considerados valores de tensão iguais a $50 \mathrm{kVp}, 70 \mathrm{kVp}$ e $100 \mathrm{kVp}$ e $60 \mathrm{~ms}, 100 \mathrm{~ms}$ e $500 \mathrm{~ms}$ para o tempo de exposição.

Para cada um dos valores nominais foram simulados quatro observações, o que corresponde ao procedimento usual de obtenção de quatro medições em um equipamento sob teste. Esses quatro valores foram simulados considerando-se a distribuição normal com média igual ao valor nominal. O desvio padrão associado a essa distribuição foi definido de tal forma que os valores simulados estivessem contidos no intervalo [LI, LS], em que LI é o limite inferior e LS é o limite superior. LI e LS foram definidos a partir de diferentes porcentagens para menos e para mais, respectivamente, do valor nominal definido. As porcentagens consideradas neste estudo foram: 0 
a $10 \%$, variando a cada $0,5 \%$, ou seja, 21 valores; de $12 \%$ a $50 \%$, variando a cada $2 \%$, ou seja, 20 valores; perfazendo, assim, um total de 41 porcentagens diferentes utilizadas nas simulações. Por exemplo, se for considerado o valor nominal igual a $20 \mathrm{kVp}$ e uma porcentagem de $10 \%$, têm-se $\mathrm{LI}=18 \mathrm{kVp}$ e $\mathrm{LS}=22 \mathrm{kVp}$.

Para garantir que aproximadamente $100 \%$ dos valores estivessem contidos no intervalo [LI, LS], o desvio padrão $(\sigma)$, foi determinado como função dos limites deste intervalo. Uma vez definidos LI e LS, pode-se determinar o valor de $\sigma$ considerando-se que, se uma variável aleatória $\mathrm{X}$ apresenta distribuição normal, ou seja, $\mathrm{X} \sim \mathrm{N}\left(\mu, \sigma^{2}\right)$, tem-se que a probabilidade $\mathrm{P}(-4 \sigma<\mathrm{X}<4 \sigma)$ $=0,9999$. Além disso, de acordo com a definição da distribuição normal padrão, tem-se que $Z=\frac{X-\mu}{\sigma}$ e $\mathrm{P}(-4<\mathrm{Z}<4)=0,9999$. Assim, considerando-se que o valor de $\mathrm{Z}$ é igual a 4 está relacionado a $L S$, tem-se que $4=\frac{L S-\mu}{\sigma}$, em que $\mu$ é igual ao valor nominal e, portanto, $\sigma=\frac{L S-\mu}{4}$

Considere o mesmo exemplo já utilizado, em que o valor tensão é igual a $20 \mathrm{kVp}$ e tem-se uma porcentagem igual a 10\%. Assim, LI e LS são iguais a 18 e 22, respectivamente. Portanto, para que $99,99 \%$ dos valores simulados pertençam ao intervalo de 18 e $22 \mathrm{kVp}$, o desvio padrão é dado por $\sigma=\frac{22-20}{4}=0,5$. Para os demais valores nominais e porcentagens, o raciocínio é o mesmo. Observe que o cálculo também pode ser efetuado considerando-se o LI. Nesse caso, temse que $\sigma=\frac{L I-\mu}{-4}$.

A porcentagem igual a zero $(0 \%)$ corresponde à obtenção de quatro medições iguais, ou seja, sem variabilidade. Nesse procedimento de simulação, tem-se que, quanto maior a porcentagem do valor nominal para estabelecer LI e LS, maior será a variabilidade das quatros observações e maior será a probabilidade de obtenção de valores mais discrepantes em relação ao valor nominal.

Diante disso, ao considerar dois tipos de equipamentos (mamógrafos e raios $\mathrm{X}$ convencionais), duas variáveis avaliadas (tensão e tempo de exposição), três valores nominais e 41 porcentagens para estabelecer LI e LS, tem-se $2 \times 2 \times 3 \times 41=492$ cenários diferentes, sendo que, de cada um dos cenários, foram simuladas 1.000 repetições, ou seja, 1.000 conjuntos com 4 valores simulados para cada cenário.

\section{RESULTADOS E DISCUSSÃO}

Inicialmente serão feitos comentários sobre as avaliações da exatidão para mamógrafos e raios $\mathrm{X}$ convencional e posteriormente para as avaliações de precisão desses mesmos equipamentos.

No contexto do controle de qualidade em radiodiagnóstico, ao avaliar a exatidão, as estatísticas utilizadas deverão estimar o quanto o valor observado da variável analisada está em concordância com o valor nominal selecionado no painel do equipamento.

As estatísticas e as respectivas tolerâncias, recomendadas pelos documentos analisados nas avaliações de exatidão em mamógrafos, estão apresentadas na Tabela 1.

Na Tabela 1, verificam-se três estatísticas diferentes para a avaliação da exatidão da tensão em mamógrafos, sendo que a Portaria Federal MS SVS 453/98 [4] e Protocolo Español 2011 [8] recomendam a mesma, com valores de tolerâncias distintas. Essas diferenças nas tolerâncias tornam a metodologia do Protocolo Español 2011 mais rigorosa, pois o início de rejeição ocorrerá antes da metodologia indicada pela Portaria Federal MS SVS 453/98 (Figura 1).

A metodologia da Portaria Federal MS SVS 453/98 [4] e do Protocolo Español 2011 [8] possuem um aspecto importante e de interesse no contexto da mamografia. Estas consideram valores de tensões fixos em suas tolerâncias e, portanto, avaliam um mesmo equipamento de forma diferente, ao alterarem os valores nominais (Figura 1). 
Tabela 1: Estatísticas e tolerâncias indicadas para avaliação de exatidão em mamógrafos pela Portaria Federal MS SVS 453/98, Guia da ANVISA, Protocolo Español 2011 e Protocolo ARCAL XLIX

\begin{tabular}{lccl}
\hline Documento & Variável avaliada & Estatística & Tolerância \\
\hline \multirow{2}{*}{ Portaria 453/98 } & Tensão no tubo & $\left(Y_{n}-Y_{i}\right)_{\max }$ & $\leq \pm 2 \mathrm{kVp}$ \\
& Tempo de exposição & $\frac{\left(Y_{n}-Y_{i}\right)_{\max }}{Y_{n}} .100$ & $\leq \pm 10 \%$ \\
\hline \multirow{2}{*}{ Guia da ANVISA } & Tensão no tubo & $\frac{Y_{n}-\bar{Y}}{Y_{n}} .100$ & $\leq \pm 5 \%$ \\
& Tempo de exposição & $\frac{Y_{n}-\bar{Y}}{Y_{n}} .100$ & $\leq \pm 10 \%$ \\
\hline Protocolo Español & Tensão no tubo & $\left(Y_{n}-Y_{i}\right)_{\max }$ & $\leq \pm 1 \mathrm{kV}$ \\
2011 & Tempo de exposição & $\mathrm{NI}$ & $\mathrm{NI}$ \\
\hline & Tensão no tubo & $\frac{\left(Y_{n}-Y_{i}\right)_{\max }}{Y_{n}} .100$ & $\leq \pm 5 \%$ \\
ARCAL XLIX & & $\frac{\left(Y_{n}-Y_{i}\right)_{\max }}{Y_{n}} .100$ & $\leq \pm 10 \%, \mathrm{t} \geq 200 \mathrm{~ms}$ \\
& Tempo de exposição & $\leq \pm 15 \%, \mathrm{t}<200 \mathrm{~ms}$ \\
\hline
\end{tabular}

$Y_{n}$ : valor nominal; $Y_{i}$ : valor da medição $i ;\left(Y_{n}-Y_{i}\right)_{\max }:$ máxima diferença entre o valor nominal e os medidos; $\bar{Y}$ : média dos valores medidos.

Essa importância deve-se ao fato da mamografia ser uma técnica que requer valores de tensão mais baixos, devido às estruturas da anatomia mamária com tecido glandular e adiposo, bem como as baixas diferenças de densidade entre o tecido mamário e os achados radiográficos. Se fossem considerados valores percentuais do nominal, como por exemplo, $10 \%$ de $40 \mathrm{kVp}$, a tolerância seria de $4 \mathrm{kVp}$, o que ampliaria a diferença de tensão e consequentemente uma produção na quantidade de raios $\mathrm{X}$ aumentada no seu valor ao quadrado. Isto porque, a quantidade de raios $\mathrm{X}$ é proporcional ao quadrado das tensões [16]. Assim, sendo estabelecido um valor fixo de $1 \mathrm{kVp}$ ou $2 \mathrm{kVp}$, essa variação de tensão representaria uma menor variação de tensão e consequente menor exposição a doses de radiação do paciente. Além disso, avaliações distintas também permitirão um maior controle nas variações de tensões, pois, conforme se observa na Figura 1, para PORTARIA MS SVS 453/98 e PROTOCOLO ESPANHOL 2011, quanto maior o valor da tensão menos tolerante a metodologia está sendo, até o momento em que todas irão rejeitar no mesmo percentual.

Ao comparar todas as metodologias, verifica-se na Figura 1 que o Guia da ANVISA é o mais tolerante, visto que o percentual de rejeição é o menor na maioria dos cenários. Tal fato deve-se à presença do valor da média dos valores observados na estatística utilizada. A média, ao considerar todos os valores observados em seu cálculo, não reflete de modo eficiente os possíveis valores discrepantes.

Na Figura 2, representam-se como as diferentes estatísticas apresentadas na Tabela 1 avaliam a exatidão para a variável tempo de exposição em mamógrafos. O Protocolo ARCAL XLIX apesar de indicar a mesma estatística que a Portaria Federal MS SVS 453/98, apresentou diferenças ao considerar valores de tolerâncias diferentes para valores de tempo menores e maiores que $200 \mathrm{~ms}$. Cabe nesse caso, chamar a atenção para a possibilidade de inconsistências. Supondo que exista algum serviço com tempo de exposição igual a $199 \mathrm{~ms}$ e $201 \mathrm{~ms}$ e obedecendo ao percentual de tolerância indicado, têm-se para 199 ms o intervalo entre $169,15 \mathrm{~ms}$ e $228,25 \mathrm{~ms}$ e para $201 \mathrm{~ms}$ o intervalo entre $180,9 \mathrm{~ms}$ e $221,1 \mathrm{~ms}$. Assim, nos intervalos entre $169,15 \mathrm{~ms}$ e $180,9 \mathrm{~ms}$ estaria conforme apenas para tensão nominal $199 \mathrm{~ms}$ e a região entre $221,1 \mathrm{~ms}$ e $228,85 \mathrm{~ms}$ estaria conforme apenas para tensão nominal igual a $201 \mathrm{~ms}$. Sendo, portanto esses intervalos um exemplo de regiões de conflito, onde ocorreriam resultados inconsistentes. 
PORTARIA MS SVS $453 / 98$

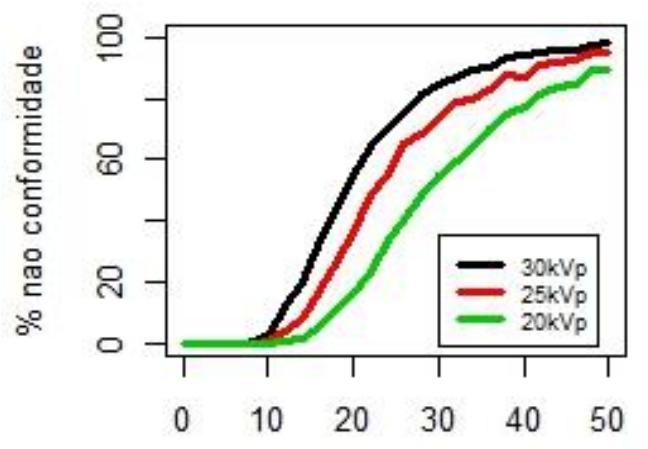

$\%$ do $\mathrm{kVp}$ que estabelece LI e LS

PROTOCOLO ESPANHOL 2011

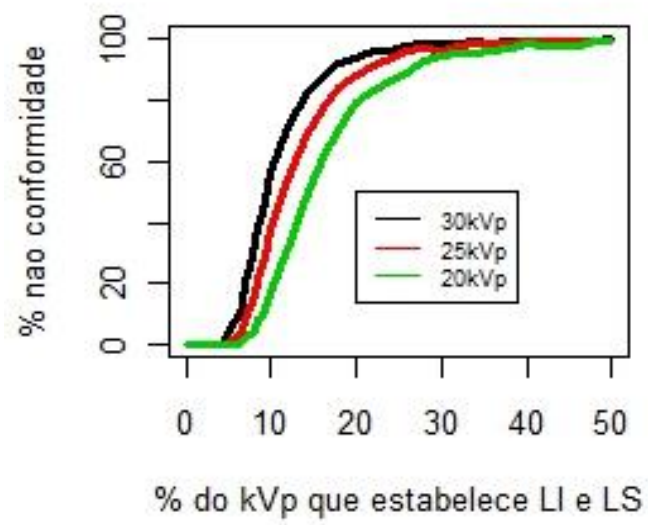

ANVISA

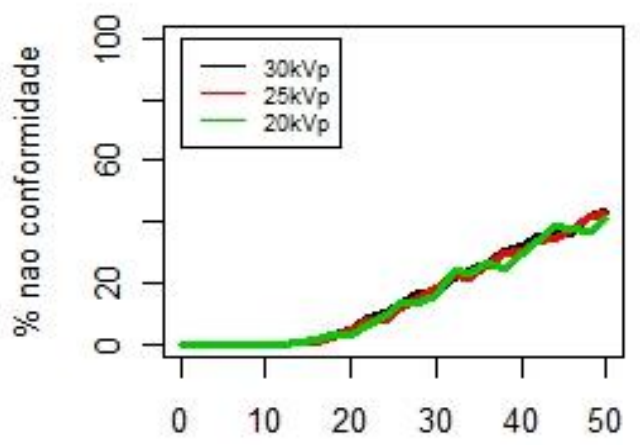

$\%$ do $\mathrm{kVp}$ que estabelece LI e LS

PROTOCOLO ARCAL XLIX

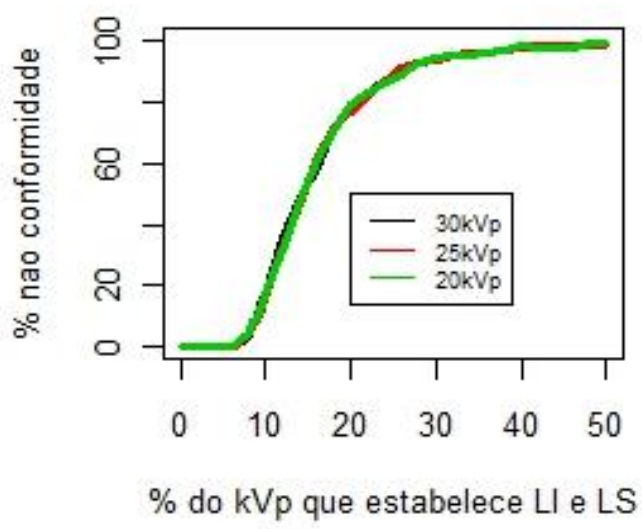

Figura 1: Porcentagem de não conformidade na avaliação da exatidão da tensão em mamógrafos conforme PORTARIA MS SVS 453/98, Guia da ANVISA, Protocolo ARCAL XLIX e Protocolo Español 2011, para valores nominais iguais a $30 \mathrm{kVp}, 25 \mathrm{kVp}$ e $20 \mathrm{kVp}$

Sendo assim, para que os critérios sejam coerentes, é necessário adotar um único percentual de tolerância independente do valor nominal.

Para os testes de exatidão em equipamentos de raios $\mathrm{X}$ convencionais, identificaram-se as expressões e tolerâncias resumidas na Tabela 2. 
PORTARIA MS SVS $453 / 98$

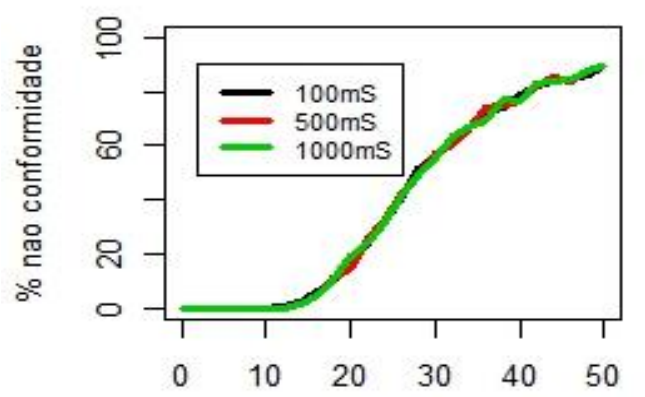

$\%$ do mS que estabelece LI e LS

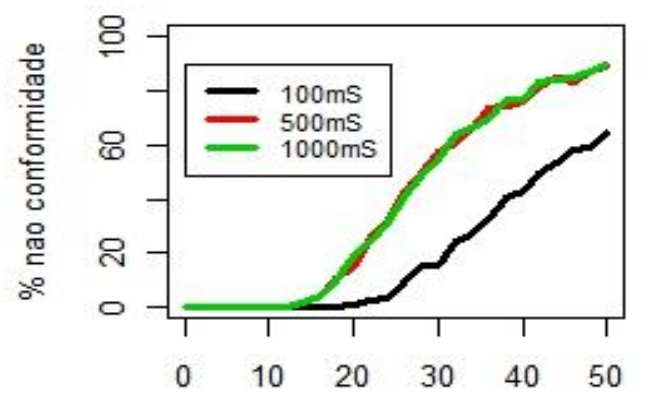

$\%$ do mS que estabelece LI e LS

Figura 2: Porcentagem de não conformidade na avaliação da exatidão do tempo de exposição em em mamógrafos conforme PORTARIA FEDERAL MS SVS 453/98, Guia da ANVISA, Protocolo ARCAL XLIX para valores nominais iguais a $100 \mathrm{~ms}, 500 \mathrm{~ms}$ e $1.000 \mathrm{~ms}$

Tabela 2: Estatísticas e tolerâncias indicadas para avaliação de exatidão em raios X convencional pela Portaria Federal MS SVS 453/98, Guia da ANVISA, Protocolo Español 2011 e Protocolo ARCAL XLIX

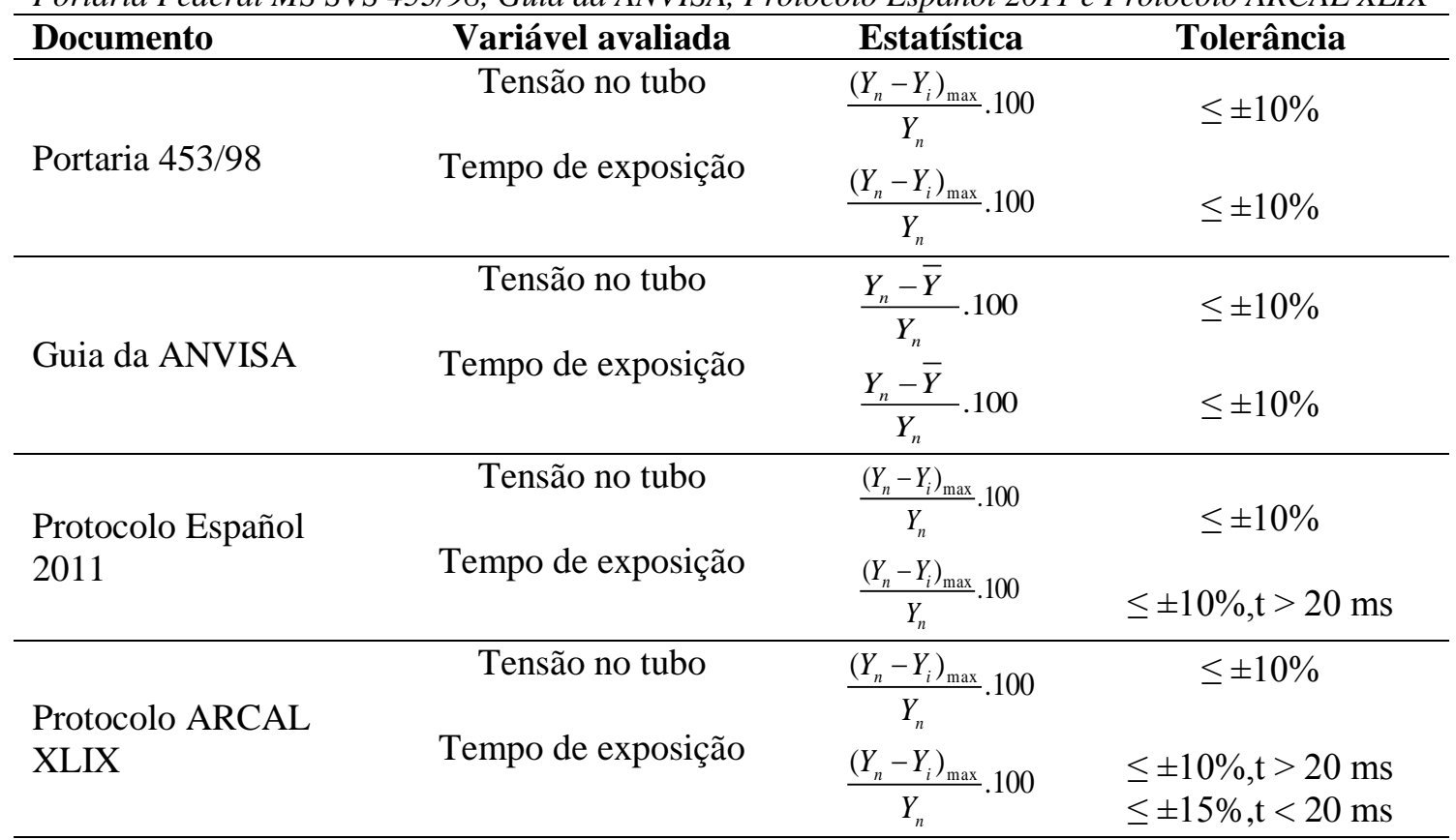

$Y_{n}:$ valor nominal; $Y_{i}:$ valor da medição $i ;\left(Y_{n}-Y_{i}\right)_{\max }:$ máxima diferença entre o valor nominal e os medidos; $\bar{Y}$ : média dos valores medidos, NI: Não indica, $t:$ tempo 
Percebe-se na Tabela 2, a presença de tolerâncias diferentes estabelecidas pelo Protocolo Español 2011 e Protocolo ARCAL XLIX, acrescentando-se o fato de não indicar para valores de tempo iguais a $20 \mathrm{~ms}$. No caso das diferentes tolerâncias, cabe a mesma discussão feita para tempo de exposição em mamógrafos. Considerando o tempo de exposição igual a $19 \mathrm{~ms}$ o intervalo de tolerância seria entre $16,15 \mathrm{~ms}$ e $21,85 \mathrm{~ms}$ e para $21 \mathrm{~ms}$ o intervalo seria entre 18,9 ms e $23,1 \mathrm{~ms}$, tendo, portanto, como regiões de conflito (ou divergências) os intervalos entre $16,85 \mathrm{~ms}$ e $18,90 \mathrm{~ms}$ e entre $21,85 \mathrm{~ms}$ e $23,1 \mathrm{~ms}$, nas quais estariam apenas em conformidade para $19 \mathrm{~ms}$ e $21 \mathrm{~ms}$ respectivamente.

Na Figura 3 e 4, verifica-se que todas as metodologias avaliam valores nominais distintos da mesma forma, nas avaliações da tensão e tempo de exposição em equipamentos de raios X. No entanto, pelos mesmos motivos já expostos nas avaliações para mamógrafos, ao comparar o método da ANVISA, percebe-se uma tolerância maior que as demais.

PORTARIA MS SVS $453 / 98$

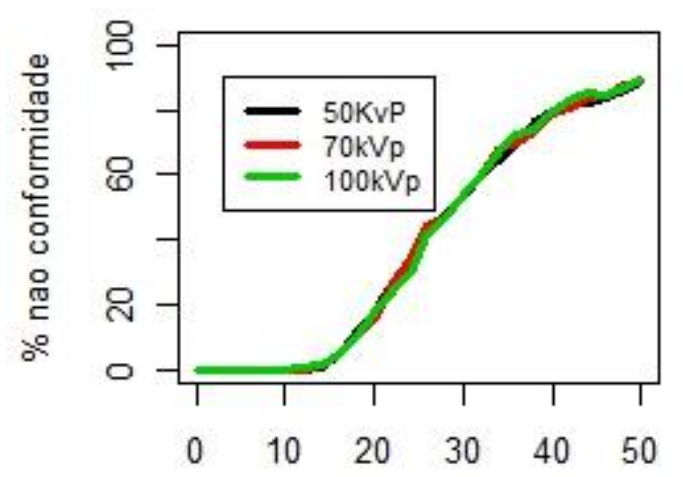

$\%$ do $\mathrm{kVp}$ que estabelece LI e LS

PROTOCOLO ESPANHOL 2011

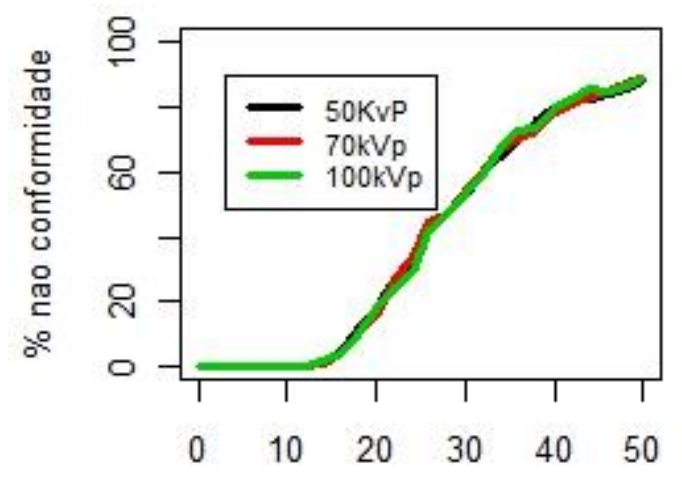

$\%$ do $\mathrm{kVp}$ que estabelece LI e LS

\section{ANVISA}

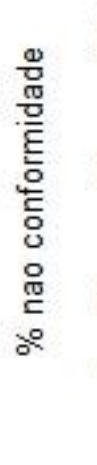

$\%$ do $\mathrm{kVp}$ que estabelece LI e LS

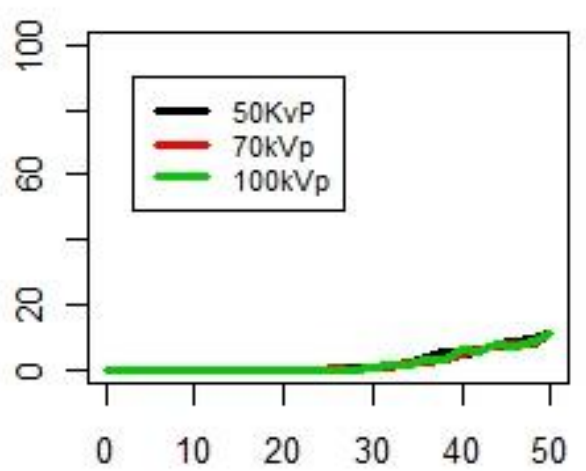

PROTOCOLO ARCAL XLIX
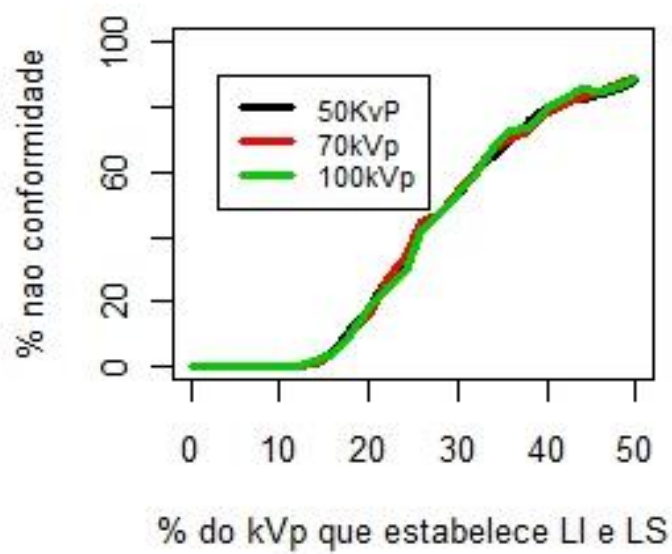

Figura 3: Porcentagem de não conformidade na avaliação da exatidão da tensão em equipamentos de raios X convencionais conforme PORTARIA FEDERAL MS SVS 453/98, Guia da ANVISA, Protocolo ARCAL XLIX para tensões nominais iguais a $50 \mathrm{kVp}$, 70kVp e $100 \mathrm{kVp}$ 
PORTARIA MS SVS $453 / 98$

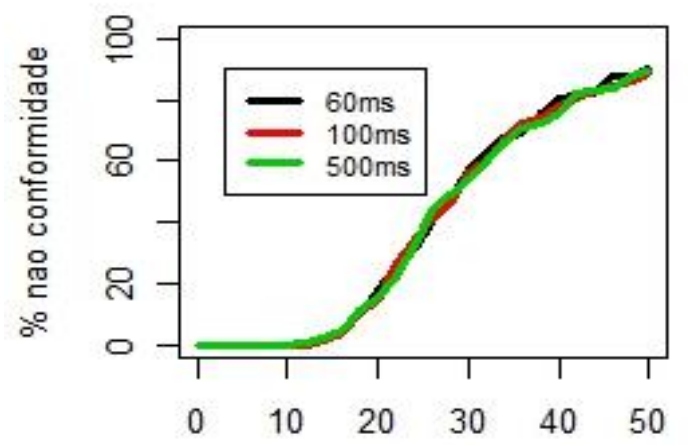

$\%$ do $\mathrm{mS}$ que estabelece LI e LS

PROTOCOLO ESPANHOL 2011

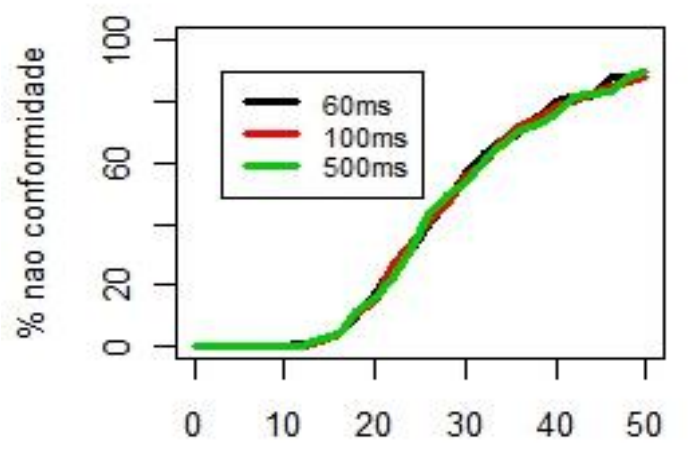

$\%$ do $\mathrm{mS}$ que estabelece LI e LS

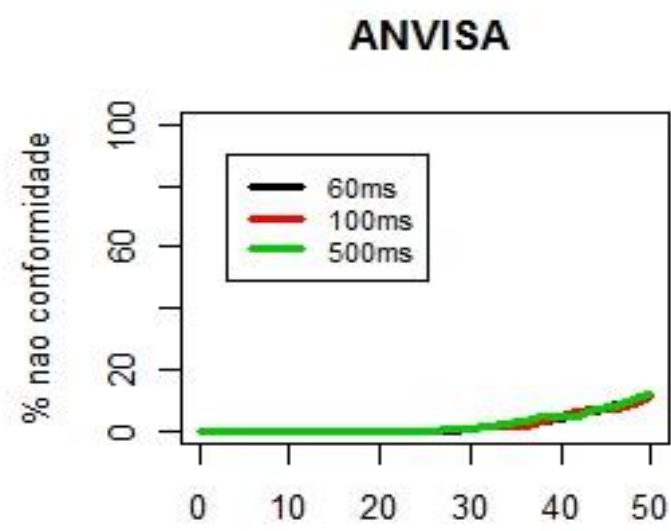

$\%$ do $\mathrm{mS}$ que estabelece LI e LS

PROTOCOLO ARCAL XLIX

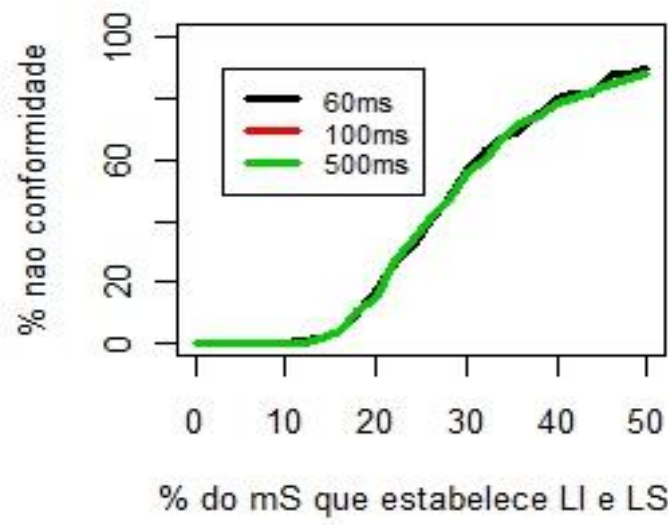

Figura 4: Porcentagem de não conformidade na avaliação da exatidão do tempo de exposição em equipamentos de raios X convencionais conforme PORTARIA FEDERAL MS SVS 453/98, Protocolo ARCAL XLIX e Protocolo Español 2011 e Guia da ANVISA para valores nominais iguais a $60 \mathrm{~ms}, 100 \mathrm{~ms}$ e $500 \mathrm{~ms}$

Nas Tabelas 3 e 4, apresentam-se as expressões consideradas nas avaliações de precisão da variável de tensão no tubo e tempo de exposição, para equipamentos de mamografia e raios $\mathrm{X}$ convencionais, respectivamente, ocorrendo algumas concordâncias com a Estatística, que considera as medidas de variabilidade tais como, variância, desvio padrão, coeficiente de variação, amplitude total, dentre outras, para caracterizar, por meio de suas estimativas, a precisão.

Estas avaliações são realizadas por meio de teste de reprodutibilidade e/ou repetitividade, que de acordo com o VIM, são precisões de medição, que consistem em grau de concordância entre indicações ou valores medidos, obtidos por medições repetidas, no mesmo objeto ou em objetos similares, diferenciados pelas condições de medição.

No contexto do controle de qualidade em radiodiagnóstico, a precisão deve verificar o quanto os valores observados obtidos do equipamento em teste estão próximos entre si.

Os documentos que não estabelecem tais testes e/ou tolerâncias, aparecem na Tabela 3 e 4 como não indica (NI). 
Tabela 3: Estatísticas e tolerâncias indicadas para avaliação de precisão em mamógrafos pela Portaria Federal MS SVS 453/98, Guia da Anvisa, Protocolo Español 2011 e Protocolo ARCAL XLIX

\begin{tabular}{|c|c|c|c|c|}
\hline Documento & Teste & Variável avaliada & Estatística & Tolerância \\
\hline \multirow{3}{*}{$\begin{array}{l}\text { Portaria federal MS } \\
\text { SVS 453/98* }\end{array}$} & \multirow{2}{*}{ RPD } & Tensão no tubo & $\frac{Y_{M}-Y_{m}}{\bar{Y}} .100$ & $\leq \pm 10 \%$ \\
\hline & & Tempo de exposição & $\frac{Y_{M}-Y_{m}}{\bar{Y}} \cdot 100$ & $\leq \pm 10 \%$ \\
\hline & RPT & $\mathrm{NI}$ & NI & ----- \\
\hline \multirow{3}{*}{ Guia da ANVISA } & \multirow[b]{2}{*}{ RPD } & Tensão no tubo & $C V=\frac{\hat{\sigma}}{\bar{Y}}$ & $<0,02$ \\
\hline & & Tempo de exposição & $\frac{Y_{M}-Y_{m}}{\left(\frac{Y_{M}+Y_{m}}{2}\right)} \cdot 100$ & $\leq \pm 10 \%$ \\
\hline & RPT & $\mathrm{NI}$ & $\mathrm{NI}$ & $\begin{array}{ll}----- \\
\end{array}$ \\
\hline \multirow[b]{2}{*}{$\begin{array}{l}\text { Protocolo } \\
\text { ESPANHOL } 2011\end{array}$} & RPD & NI & NI & ------ \\
\hline & RPT & $\begin{array}{c}\text { Tensão no tubo } \\
\text { Tempo de exposição }\end{array}$ & $\begin{array}{c}\left|Y_{i}-\bar{Y}\right|_{\max } \\
\text { NI }\end{array}$ & $\leq \pm 0,5 \mathrm{kVp}$ \\
\hline \multirow{3}{*}{$\begin{array}{l}\text { Protocolo ARCAL } \\
\text { XLIX }\end{array}$} & RPD & NI & $\begin{array}{r}\mathrm{NI} \\
Y_{M}-Y_{m}\end{array}$ & ----- \\
\hline & \multirow{2}{*}{ RPT } & Tensão no tubo & $\left(\frac{Y_{M}+Y_{m}}{2}\right) .100$ & $\leq \pm 2 \%$ \\
\hline & & Tempo de exposição & $\frac{Y_{M}-Y_{m}}{\left(\frac{Y_{M}+Y_{m}}{2}\right)}$ & $\leq \pm 10 \%$ \\
\hline
\end{tabular}

$\overline{Y_{M}}:$ Maior valor da série de observações, RPD:Reprodutibilidade, RPT:Repetitividade; $\quad Y_{m}:$ Menor valor da série de observações. $\bar{Y}$ : média dos valores medidos, $\hat{\sigma}$ : desvio padrão amostral, $\left|Y_{i}-\bar{Y}\right|_{\max }$ :desvio absoluto máximo, $Y_{M}-Y_{m}:$ Amplitude total, $\frac{Y_{M}+Y_{m}}{2}:$ Amplitude média, NI: Não indica.

A precisão para tempo de exposição em mamógrafo está sendo verificada, conforme Tabela 3, por duas estatísticas diferentes. Porém as tolerâncias são as mesmas. Essas diferenças não são percebidas no percentual de rejeição, como mostra a Figura 5, indicando que as avaliações são coincidentes, independente dos valores nominais.

Ainda na Tabela 3, verifica-se que existem quatro estatísticas diferentes para avaliar precisão da tensão e no tempo de exposição, conforme o documento considerado. Essas conduzem a diferentes formas de avaliar a precisão, como pode ser visto na Figura 6. Ao considerar tolerâncias distintas, as metodologias indicadas pela Portaria Federal MS SVS 453/98 e Protocolo ARCAL XLIX apresentaram diferenças nos resultados. A Portaria Federal MS SVS 453/98, por indicar um percentual maior, mostrou-se mais tolerante. 
PORTARIA MS SVS $453 / 98$

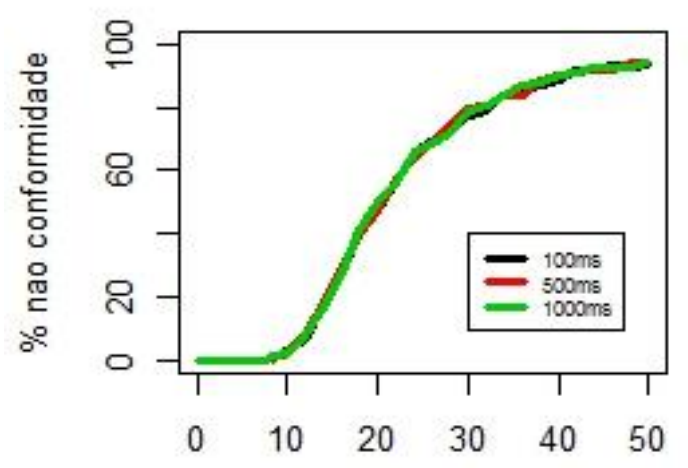

$\%$ do ms que estabelece LI e LS

\section{PROTOCOLO ARCAL XLIX}

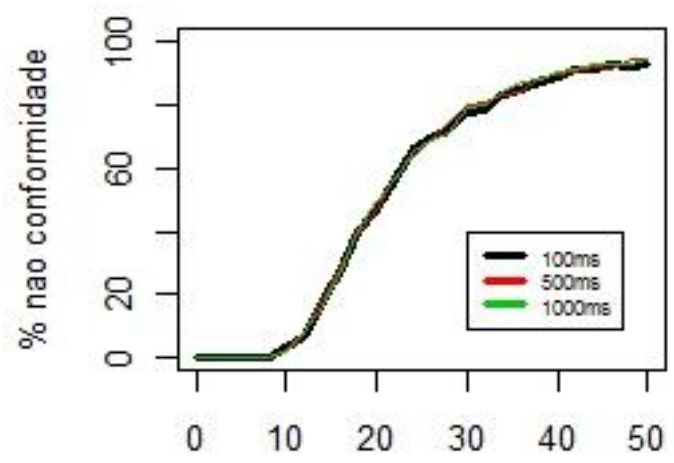

$\%$ do ms que estabelece LI e LS
ANVISA

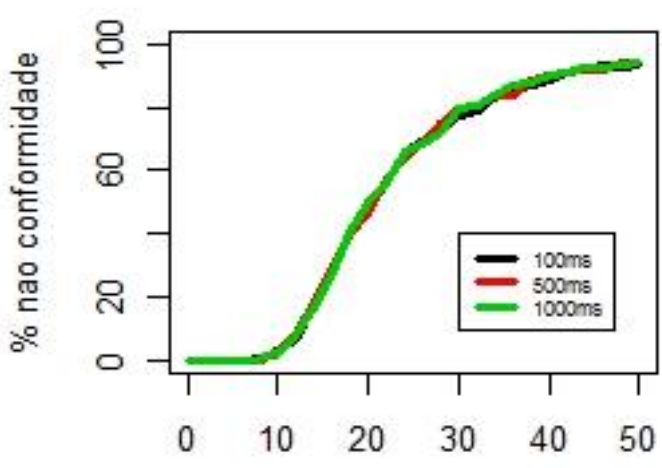

$\%$ do $\mathrm{ms}$ que estabelece LI e LS

Figura 5: Porcentagem de não conformidade na avaliação da precisão do tempo de exposição em mamógrafos conforme PORTARIA FEDERAL MS SVS 453/98, Guia da ANVISA e Protocolo ARCAL XLIX e para valores nominais iguais a $100 \mathrm{~ms}, 500 \mathrm{~ms}$ e $1.000 \mathrm{~ms}$

Outra observação pode ser feita na metodologia do Protocolo Español 2011, cujas curvas para $20 \mathrm{kVp}, 25 \mathrm{kVp}$ e $30 \mathrm{kVp}$ apresentam comportamentos diferentes. Portanto, apresentam resultados diferentes nas porcentagens de rejeição. Isto indica que tal metodologia apresenta diferentes resultados com a alteração no valor nominal.

Na Tabela 4, apresenta-se um resumo das recomendações para avaliar a precisão da tensão e para o tempo de exposição, nos equipamentos de raios $\mathrm{X}$ convencionais.

Observa-se na Figura 7 que as metodologias estabelecidas pela Portaria Federal MS SVS 453/98, Guia da ANVISA e Protocolo ARCAL XLIX, não altera o percentual de rejeição quando se altera o valor nominal da tensão. O uso do coeficiente de variação estabelecido pelo Protocolo Español 2011 demonstra-se um pouco menos rigoroso que os demais. Mesma consideração pode ser feita para a variável tempo de exposição em equipamentos de raios $\mathrm{X}$ convencionais (Figura 8).

Porém observa-se nesse último caso, uma diferença bem maior com relação ao rigor das metodologias na detecção de equipamentos fora da especificação. O Protocolo Español 2011 mostrou-se bem menos rigoroso que os demais.

Além das inconsistências apontadas anteriormente, outros problemas também foram detectados, como por exemplo, a falta de formalidade nas definições das tolerâncias. Pode-se 
observar na tolerância indicada pelo Protocolo Español 2011 na Tabela 4, ao indicar $0.5 \mathrm{kVp}$ como tolerância. Utilizar os sinais não faz sentido, uma vez que a estatística fornece valores absolutos e, portanto, deve-se utilizar apenas uma tolerância positiva.

A Portaria Federal MS SVS 453/98 estabelece teste de reprodutibilidade apenas para taxa de kerma no ar e do sistema automático de exposição. Sendo assim, este trabalho adotou a mesma expressão e tolerância para avaliar as variáveis tensão e tempo de exposição.

Segue na Tabela 5, um resumo das estatísticas recomendadas como a mais adequada para as avaliações de exatidão e precisão das variáveis como tempo de exposição e tensão no tubo no contexto do controle de qualidade em radiodiagnostico.

PORTARIA MS SVS 453/98

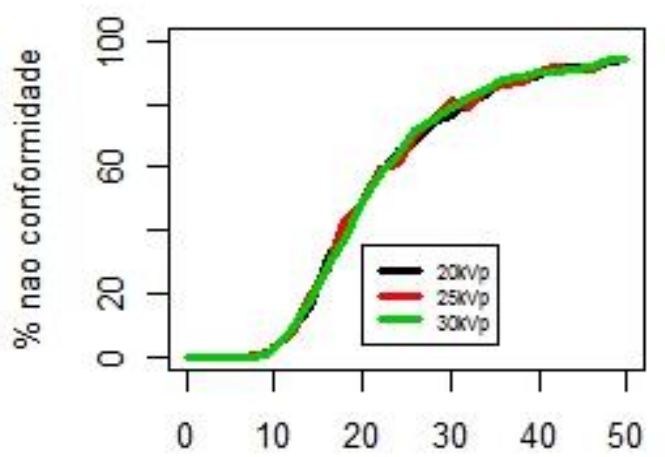

$\%$ do $\mathrm{kVp}$ que estabelece LI e LS

PROTOCOLO ESPANHOL 2011

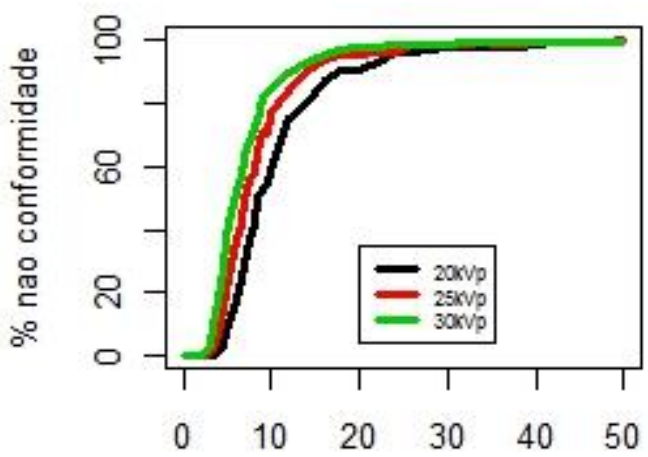

$\%$ do $\mathrm{kVp}$ que estabelece LI e LS
ANVISA

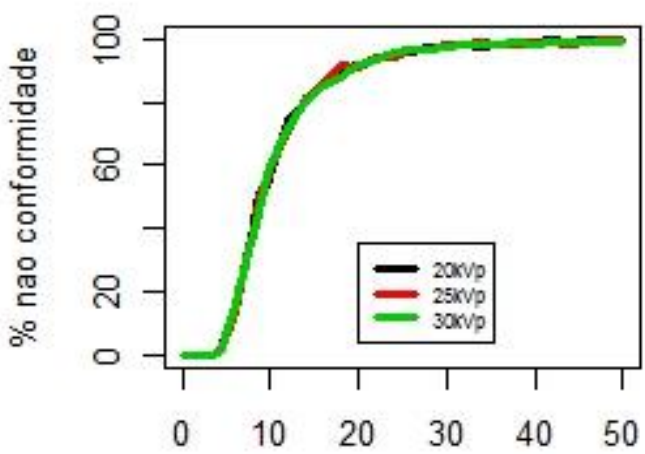

$\%$ do $\mathrm{kVp}$ que estabelece LI e LS

PROTOCOLO ARCAL XLIX

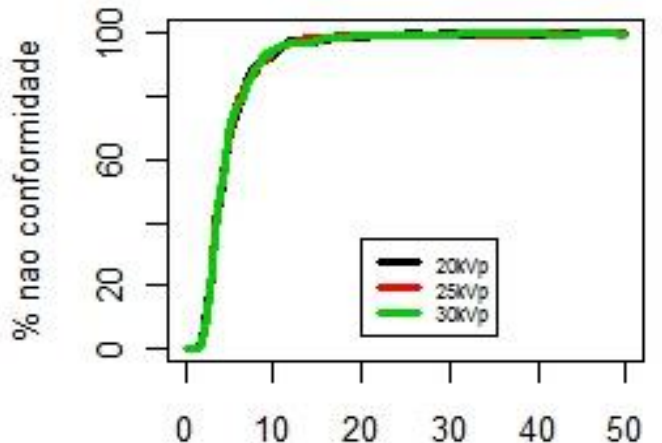

$\%$ do $\mathrm{kVp}$ que estabelece LI e LS

Figura 6: Porcentagem de não conformidade na avaliação da precisão da tensão em mamógrafos conforme PORTARIA FEDERAL MS SVS 453/98, Guia da ANVISA, Protocolo Español 2011 e Protocolo ARCAL XLIX para valores nominais iguais a $20 \mathrm{kVp}$, $25 \mathrm{kVp}$ e $30 \mathrm{kVp}$ 
PORTARIA MS SVS $453 / 98$

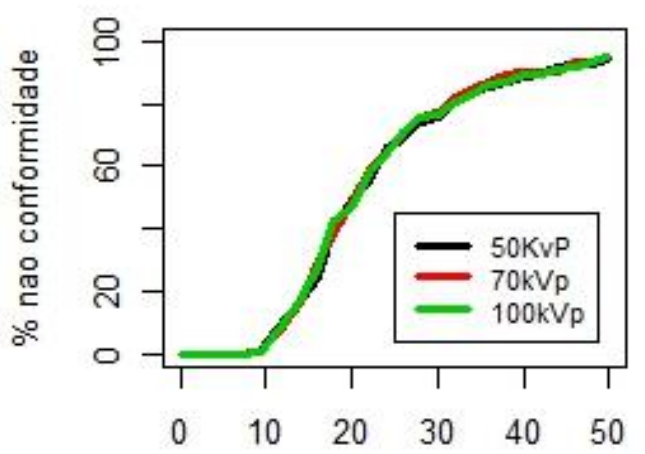

$\%$ do $\mathrm{kVp}$ que estabelece LI e LS

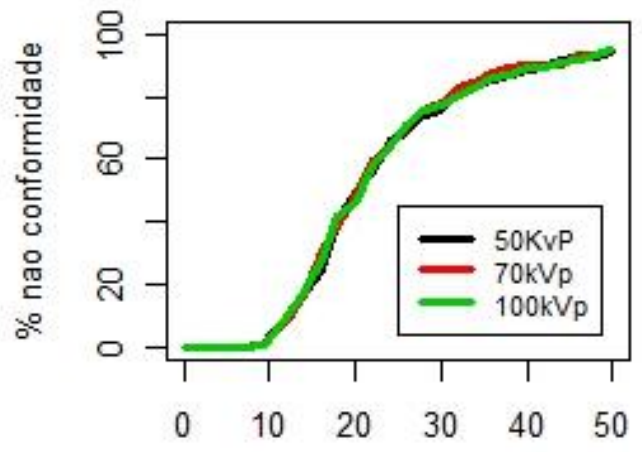

$\%$ do $\mathrm{kVp}$ que estabelece LI e LS

PROTOCOLO ESPANHOL 2011
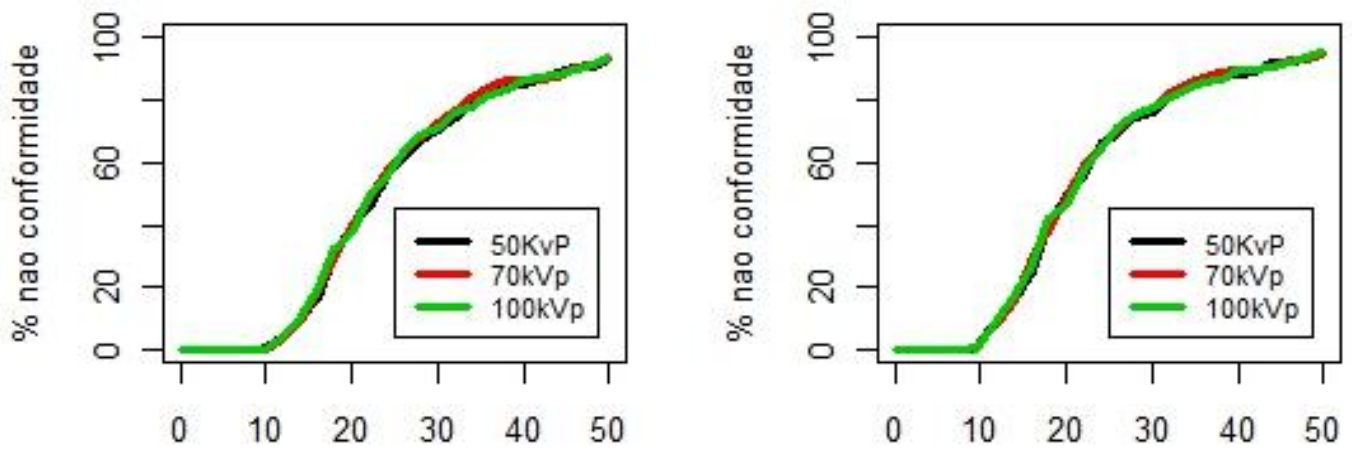

$\%$ do $\mathrm{kVp}$ que estabelece LI e LS

PROTOCOLO ARCAL XLIX

\section{ANVISA}

$\%$ do $k V p$ que estabelece LI e LS

Figura 7: Porcentagem de não conformidade na avaliação da precisão da tensão em equipamentos de raios X convencionais conforme PORTARIA FEDERAL MS SVS 453/98, Guia da ANVISA, Protocolo

Español 2011 e Protocolo ARCAL XLIX para valores nominais iguais a 50kVp, 70kVpe 100kVp 
Tabela 4: Estatísticas e tolerâncias indicadas para avaliação de precisão para raios X convencional pela Portaria Federal MS SVS 453/98, Guia da ANVISA, Protocolo Español 2011 e Protocolo ARCAL XLIX

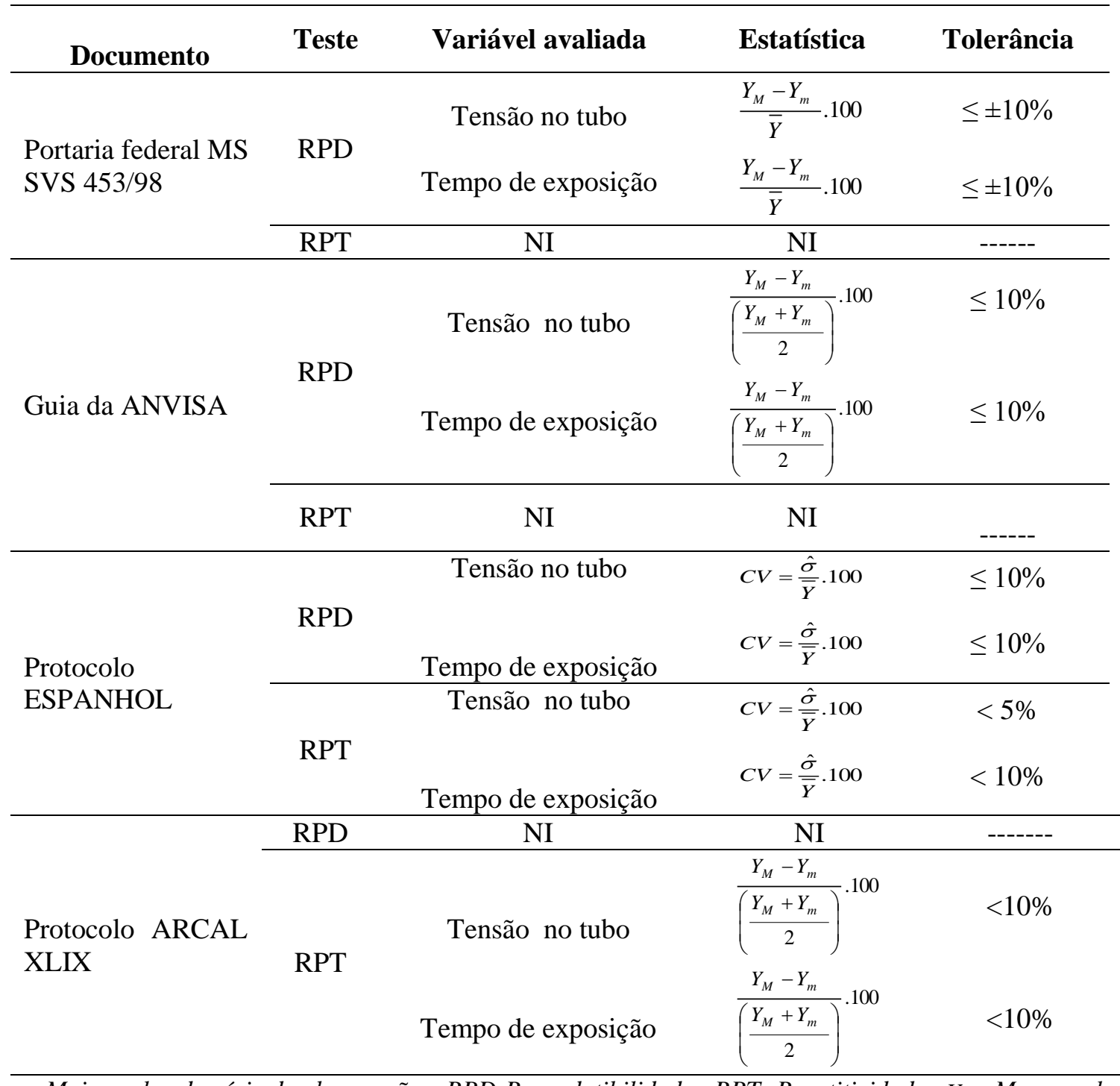

$\overline{Y_{M}}:$ Maior valor da série de observações; RPD:Reprodutibilidade, RPT: Repetitividade; $Y_{m}:$ Menor valor da série de observações NI: Não indica, $\bar{Y}$ : média dos valores medidos, $\hat{\sigma}:$ desvio padrão amostral, $Y_{M}-Y_{m}:$ Amplitude total, $\frac{Y_{M}+Y_{m}}{2}:$ Amplitude média 
PORTARIA MS SVS 453/98

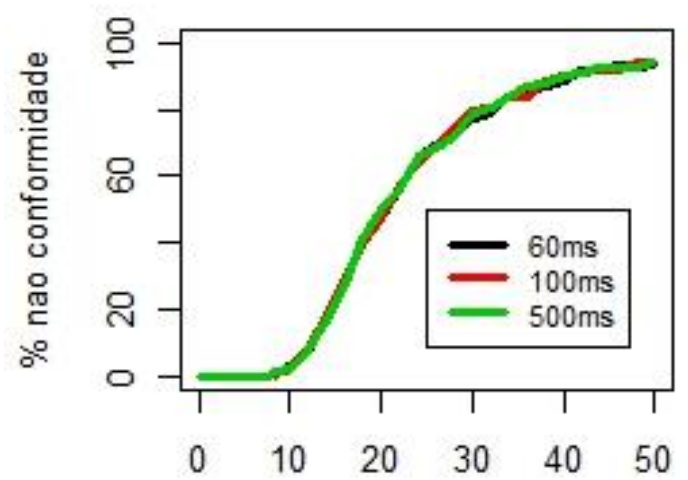

$\%$ ms que estabelece LI e LS

PROTOCOLO ESPANHOL 2011

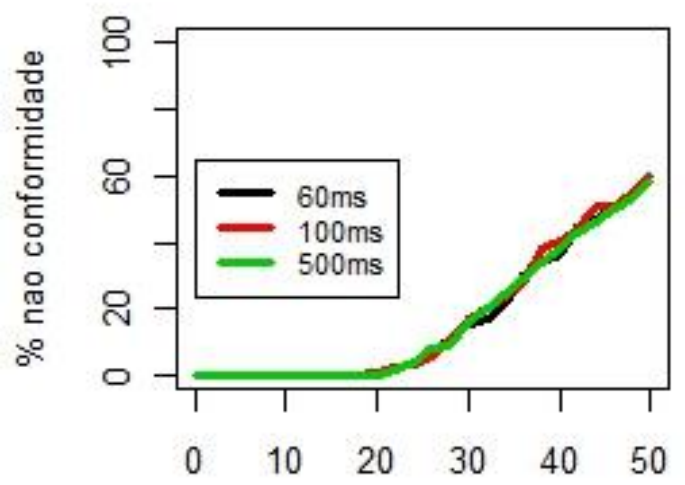

$\%$ ms que estabelece LI e LS
ANVISA

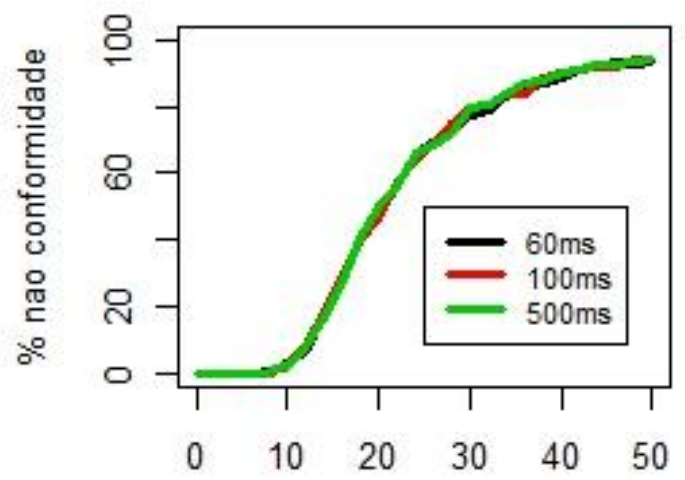

$\%$ ms que estabelece LI e LS

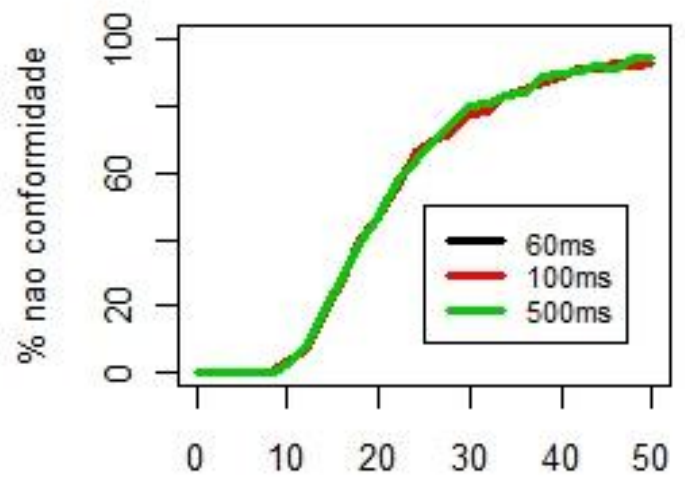

$\%$ ms que estabelece LI e LS

Figura 8: Porcentagem de não conformidade na avaliação da precisão do tempo de exposição em equipamentos de raios X convencionais conforme PORTARIA FEDERAL MS SVS 453/98, Guia da ANVISA, Protocolo Español 2011 e Protocolo ARCAL XLIX para valores nominais iguais a $60 \mathrm{~ms}, 100 \mathrm{~ms}$ e $500 \mathrm{~ms}$ 
Tabela 5: Estatísticas recomendadas como as mais adequadas para a avaliação da exatidão e da precisão da tensão do tubo e tempo de exposição em equipamentos para Radiodiagnóstico

\begin{tabular}{|c|c|c|}
\hline & Mamógrafos & \\
\hline Teste & Variável avaliada & Estatística recomendada \\
\hline \multirow{3}{*}{ Exatidão } & Tensão no tubo & $\left(Y_{n}-Y_{i}\right)_{\max }$ \\
\hline & & $\left(Y_{n}-Y_{i}\right)_{\max } 100$ \\
\hline & Tempo de exposição & $Y_{n} .100$ \\
\hline \multirow{4}{*}{ Precisão } & Tensão no tubo & $\left|Y_{i}-\bar{Y}\right|$ \\
\hline & & $Y_{M}-Y_{m}$ \\
\hline & Tempo de exposição & $\overline{\left(Y_{M}+Y_{m}\right)} \cdot 100$ \\
\hline & & 2 \\
\hline \multicolumn{3}{|c|}{ Raios X Convencionais } \\
\hline \multirow{4}{*}{ Exatidão } & Tensão no tubo & $\left(Y_{n}-Y_{i}\right)_{\max } 100$ \\
\hline & & $Y_{n}$ \\
\hline & & $\left(Y_{n}-Y_{i}\right)_{\max } 100$ \\
\hline & Tempo de exposição & $Y_{n} .100$ \\
\hline \multirow{6}{*}{ Precisão } & Tensão no tubo & $Y_{M}-Y_{m}$ \\
\hline & & $\overline{Y_{M}+Y_{m}}$ \\
\hline & & 2 \\
\hline & \multirow{3}{*}{ Tempo de exposição } & $Y_{M}-Y_{m}$ \\
\hline & & $\overline{\left(Y_{M}+Y_{m}\right)} \cdot 100$ \\
\hline & & $(2)$ \\
\hline
\end{tabular}

$Y_{M}:$ Maior valor da série de observações, NI: Não indica, $Y_{m}:$ Menor valor da série de observações $\bar{Y}$ : média dos valores medidos, $Y_{M}-Y_{m}:$ Amplitude total, $\frac{Y_{M}+Y_{m}}{2}:$ Amplitude média

\section{CONSIDERAÇÕES FINAIS}

Uma conclusão deve ser apresentada com as principais contribuições do estudo. As diferentes estatísticas utilizadas para estimar a exatidão e precisão em todos os documentos analisados são adequadas de acordo com as definições do VIM e as teorias estatísticas. No contexto do controle de qualidade para radiodiagnóstico, deseja-se detectar possíveis discrepâncias ou valores extremos ocorridos na série de observações, sejam elas em relação ao valor nominal, quando se avalia a exatidão, sejam elas em relação aos valores observados, quando se avalia a precisão. Nesse sentido, essa detecção fica comprometida em expressões que utilizam média em seus cálculos, pois, apesar de valores extremos exercerem grande influência sobre o valor da média, esta não reflete de modo eficiente os possíveis valores discrepantes.

Como consequência, as expressões, que avaliam exatidão ou precisão, ao utilizar a média das observações em seus cálculos, podem não refletir valores discrepantes de forma mais sensível que outras estatísticas, como a amplitude total, por exemplo, aplicadas nas avaliações de precisão, que faz uso de apenas duas observações. Portanto, estatísticas que consideram a média dos valores observados em seus cálculos mostram-se, de forma geral, menos adequadas nas avaliações de controle de qualidade em radiodiagnóstico.

Uma exceção para o uso da média ocorre no caso da avaliação da precisão da tensão em mamógrafos. Neste caso, devido às características do equipamento, em que é necessário um maior controle na variável tensão, a estatística recomendada pelo Protocolo Español 2011, por avaliar de forma diferenciada valores nominais distintos, mesmo fazendo uso da média, é a mais adequada. 
Sendo assim, o uso de expressões que consideram a amplitude total e desvios absolutos máximos dos valores observados são mais rigorosas no sentido de apontarem com maior sensibilidade a presença de valores discrepantes, os quais estão relacionados à aplicação de doses desnecessárias ao paciente e influenciam na qualidade da imagem.

Os valores indicados para tolerâncias têm reflexo direto na metodologia, tornando-a mais ou menos rigorosa. $\mathrm{O}$ uso de diferentes valores de tolerância para uma mesma variável avaliada conduziu a regiões ou intervalos de conflitos, gerando inconsistências de resultados para o mesmo equipamento em teste. Como forma de padronização no âmbito nacional, recomenda-se aplicar as tolerâncias estabelecidas pela Portaria Federal MS SVS 453/98.

Com base nas características dos serviços, nas definições metrológicas e de conceitos estatísticos e tomando como principio básico do controle de qualidade a diminuição de doses de radiação ao paciente e imagens com qualidade diagnóstica, recomendam-se, entre as propostas pelos documentos analisados, as estatísticas apresentadas na Tabela 5 como as mais adequadas para as avaliações de exatidão e precisão para mamógrafos e raios $\mathrm{X}$ convencionais.

\section{REFERÊNCIAS BIBLIOGRÁFICAS}

1. World Health Organization. Quality assurance in radiology. Geneva: WHO; 1982. 64 p.

2. Organización Panaamericana de la Salud- OPAS. Organización, desarrollo, garantía de calidad y radioprotección en los servicios de radiología: imaginología y radioterapia. Washington: OPAS; 1997. $328 \mathrm{p}$.

3. São Paulo. Secretaria de Estado da Saúde. Resolução SS 625/94, de 14 de dezembro de 1994. Norma técnica que dispõe sobre o uso, posse e armazenamento de fontes de radiação ionizante no âmbito do Estado de São Paulo. Diário Oficial do Estado, São Paulo, 15 dez. 1994. Seção 1, p. 13-16. Disponível em:<http://www.ribeiraopreto.sp.gov.br/ssaude/vigilancia/vigsan/e-a-saude/resolucao_625_ 19941214. pdf $>$. Acesso em: 10 dez. 2013.

4. Brasil. Portaria $\mathrm{n}^{\circ}$ 453. Diretrizes de proteção radiológica em radiodiagnóstico médico e odontológico. Diário Oficial [da] República Federativa do Brasil. Brasília: DOU; n. 102, 1998. p. 1, Seção 1.

5. Furquim TAC, Costa PR. Garantia de qualidade em radiologia diagnóstica. Rev Bras Física Médica. 2009 Out;3(1):91-99.

6. Brasil. Resolução ${ }^{\circ}$ 64, de 4 de abril de 2003. Determina a publicação de orientação técnica sobre Guia de Procedimentos para Segurança e Qualidade da Imagem em Radiodiagnóstico Médico. Brasília: DOU; 2003. $44 \mathrm{p}$.

7. Brasil. Radiodiagnóstico médico: segurança e desempenho de equipamentos. Brasília:DOU; 2005. 104 p.

8. Sociedad Española de Física Médica; Sociedad Española de Protección Radiológica. Protocolo español de control de calidad em radiodiagnóstico: aspectos técnicos. Madrid: Edicomplet; 2011. 321 p.

9. International Commission on Radiological Protection- IAEA. Acuerdo regional de cooperaçión para la promocion de la ciencia y tecnología nuclear en Latino América y el Caribe: protocolos de control de calidad en radiodiagnostico: IAEA/ARCAL XLIX. Vienna: IAEA; 2001. 100 p.

10. Campos PM, Muniz JE, Oliveira MS, Ferreira DF. Estimativa da repetitividade e da reprodutividade de laboratórios e sua aplicação no controle de qualidade de análise do solo. Cienc Agrotec. 1999 abr/jun; 23(2):404-409.

11. Chui QSH, Barros CB de, Silva TD da. Parâmetros $r$ e R obtidos de programas interlaboratorial: como usá-los. Quím Nova. 2009 32(8):2209-2213, doi:10.1590/S0100-40422009000800037.

12. Kataoka VY, Carvalho MLM, Oliveira MS, Caldeira CM. Validação de metodologia para o teste de germinação em sementes de nabo forrageiro. Rev Bras de Sementes 2011 33(1):69-79, doi: 10.1590/S0101-31222011000100008.

13. Instituto Nacional de Metrologia, Qualidade e Tecnologia- INMETRO. Vocabulário internacional de termos fundamentais e gerais de metrologia. 2. ed. Brasília: SENAI/DN; 2000. 78 p.

14. Ferreira DF. Estatística básica. Lavras: UFLA; 2005. 664 p.

15. R Development Core Team. R: a language and environment for statistical computing. Vienna: $\mathrm{R}$ Foundation for Statistical Computing, 2012. Disponível em: 〈http://www.r-project.org〉. Acesso em: 10 dez. 2012.

16. Bushong SC. Ciência radiológica para tecnólogos: física, biologia e proteção. Rio de Janeiro: Elsevier; 2010. $709 \mathrm{p}$. 